\title{
3D Electro-optical Simulations for Improving the Photon Detection Probability of SPAD Implemented in FD-SOI CMOS Technology
}

\author{
S. Gao \\ Univ Lyon \\ INSA Lyon, CNRS, INL, UMR5270 \\ Villeurbanne, France \\ shaochen.gao@insa-lyon.fr \\ F. Mandorlo \\ Univ Lyon \\ INSA Lyon, CNRS, INL, UMR5270 \\ Villeurbanne, France \\ fabien.mandorlo@insa-lyon.fr \\ F. Calmon \\ Univ Lyon \\ INSA Lyon, CNRS, INL, UMR5270 \\ Villeurbanne, France \\ francis.calmon@insa-lyon.fr
}

\author{
D. Issartel \\ Univ Lyon \\ INSA Lyon, CNRS, INL, UMR5270 \\ Villeurbanne, France \\ dylan.issartel@insa-lyon.fr \\ D. Golanski \\ STMicroelectronics \\ Crolles, France \\ dominik.golanski@st.com
}

\author{
R. Orobtchouk \\ Univ Lyon \\ INSA Lyon, CNRS, INL, UMR5270 \\ Villeurbanne, France \\ regis.orobtchouk@insa-lyon.fr \\ A. Cathelin \\ STMicroelectronics \\ Crolles, France \\ andreia.cathelin@st.com
}

\begin{abstract}
In this article, a 3D electro-optical simulation method is presented in order to estimate the Photon Detection Probability (PDP) of Single-Photon Avalanche Diodes (SPAD). The efficiency of the proposed simulation flow is demonstrated through a complete study aimed at improving the PDP of a SPAD implemented in $28 \mathrm{~nm}$ Fully Depleted Silicon-On-Insulator (FD-SOI) CMOS technology using a light-trapping approach (thanks to the patterning of Shallow Trench Insolation - STI layer). Simulation shows an increase of PDP spectrum of over $50 \%$ at wavelengths of $400-550 \mathrm{~nm}$ and $750-1000 \mathrm{~nm}$ and of $10-15 \%$ at the wavelengths of 550 $750 \mathrm{~nm}$, compared to a reference SPAD without any nanostructuration.
\end{abstract}

Keywords - SPAD; 28nm FD-SOI CMOS; Photon Detection Probability PDP; TCAD simulation; light-trapping

\section{I.INTRODUCTION}

Single-Photon Avalanche Diodes (SPAD) or Geigermode Avalanche Diodes have been a very active research topic for the last few years due to their relatively simple structure making them easy to be implemented in CMOS technologies and also due to their sub-nanosecond response time and high light sensitivity [1]. The SPAD devices are frequently used for several purposes: Time-Of-Flight (TOF) [2] for 3D imaging and LIDAR applications, charged particle detection for medical ad high energy physics applications [3], Fluorescence Lifetime Imaging Microscopy (FLIM) for biological applications [4]. SPAD devices have been implemented in 28nm Fully Depleted Silicon-On-Insulator (FD-SOI) CMOS technology, which allows intrinsic 3D pixel [5] and indirect sensing of SPAD avalanche events [6]. In this technology, the SPAD is implemented below the buried oxide (BOX) layer and the electronic logic circuits are inserted in the thin silicon layer on top of the BOX layer, which provides an intrinsic 3D stack and a much higher fill-factor.

One of the key figures of merit of SPAD devices is the Photon Detection Probability $(P D P)$ leading to important research studies. PDP represents the SPAD devices' sensitivity and is defined as the probability of an absorbed incident photon to generate an avalanche event. $P D P$ is affected by several factors: wavelength, applied voltage, photogeneration rate, electric field, doping levels. In order to improve the $P D P$, authors in [7]-[9] respectively employ the following approaches: i) an optimized SPAD architecture with charge-focusing, ii) an increase of lightmaterial interaction thanks to nanostructuration, and iii) a germanium cavity as an absorption layer. In this study, we propose an efficient 3D electro-optical simulation method to estimate the PDP. Then, using the proposed electrooptical simulation flow, we implement the light-trapping approach to improve the PDP of SPAD devices implemented in 28nm FD-SOI CMOS technology.

In section II, the PDP estimation method, the simulation flow and an analyse of electrical behaviours of SPAD structure are described, while in section III, the simulation methodology with the light-trapping approach is presented. Simulation results of proposed electro-optical simulation flow are reported and discussed in section IV.

\section{Photon Detection Probabily Simulation METHOD}

In order to estimate the PDP of SPAD devices, we integrated the product of the Avalanche Triggering Probability $(A T P)$ and the electron-hole photogeneration rate $(G)$ over the active volume $(V)$ of the SPAD. Then we divided by the incident photon flux $\Phi_{\text {photons }}$ (number per second) at the considered wavelength $\lambda$ :

$P D P(\lambda)=\frac{1}{\Phi_{\text {photons }}(\lambda)} \iiint A T P(x, y, z) * G(x, y, z, \lambda) d V$

The simulation flow was then divided into two parts: electrical part and optical part. We performed the electrical and optical simulations with Synopsys Sentaurus and Lumerical FDTD respectively to obtain data for both the electrical data: $A T P$, electric field $(E)$, carrier mobility $(\mu)$ and optical data: the photogeneration rate $(G)$. The 
electrical and optical data are combined and postprocessed within an external Matlab routine, as shown in Fig. 1.

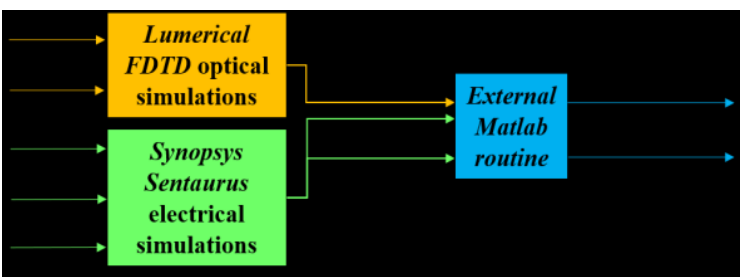

Figure 1 Block diagram of Photon Detection Probability estimation

We considered that the SPAD was composed of the Space Charge Region - SCR (as multiplication region) and two quasi-neutral regions, one p-type doped on top of the SCR and the other n-type doped at the bottom of the SCR. We calculated the limits of the active region (where photogenerated carriers effectively lead to an avalanche event) by investigating the electric field distribution across the structure and also the ratio of drift and diffusion velocities of minority carriers in each quasi-neutral region to consider the contribution of these regions to the $P D P$. Fig. 2 and 3 illustrate the profiles of electric field and ratio of drift and diffusion velocities of minority carriers respectively at the $\mathrm{p}$-side and the n-side limits of SCR with an excess voltage of $1.5 \mathrm{~V}$ ( $15 \%$ of the breakdown voltage).

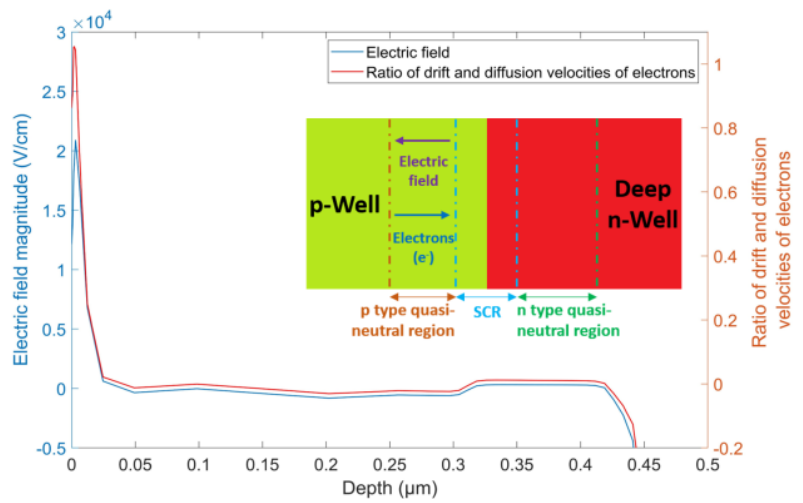

Figure 2 Profiles of electric filed and ratio of drift and diffusion velocities of electrons at the p-side limit of SCR

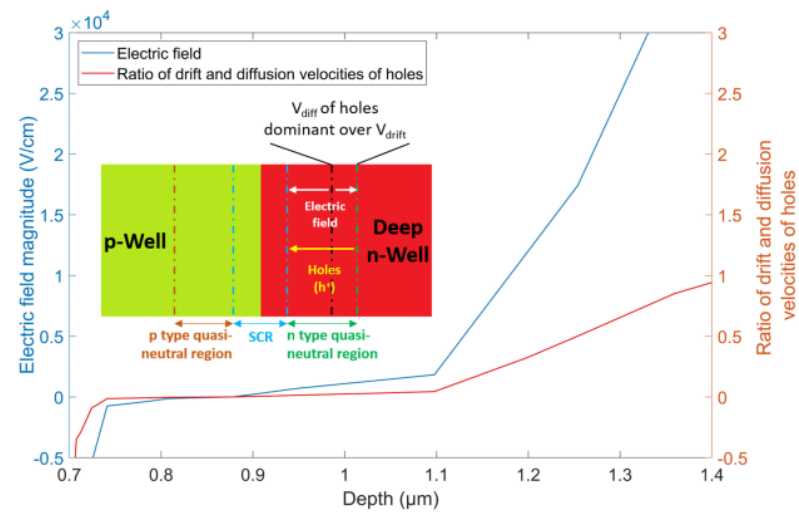

Figure 3 Profiles of electric filed and ratio of drift and diffusion velocities of holes at the n-side limit of SCR

We considered that the beginning of p-type quasi-neutral region is given by the direction of electrical field that was favourable for electrons and the beginning of SCR (end of p-type quasi-neutral region) corresponds to the electric field magnitude was below the threshold of $-10^{5} \mathrm{~V} / \mathrm{cm}$. The end of SCR (beginning of n-type quasi-neutral region) was where the electric filed surpassed the threshold. For the end of the n-type quasi-neutral region, the diffusion velocity ( $V_{\text {diff }}$ ) of holes is dominant over the drift velocity $\left(V_{\text {drift }}\right)$ of holes in some areas and we considered that when the ratio $V_{\text {drift }} / V_{\text {diff }}$ was above 0.5 , the n-type quasineutral region ended.

The electrical simulations were 2D cylindrical symmetry whereas the optical simulations were 3D (with adapted limit conditions linked to spatial period). The external Matlab was able to extrapolate the 2D electrical data according to the cylindrical symmetry and to create 3D matrix of electrical data. It was thus able to combine $2 \mathrm{D}$ electrical and 3D optical data to calculate de PDP.

\section{Simulation Methodology}

A cross section of the investigated SPAD implemented in 28nm FD-SOI CMOS technology is shown in Fig. 4. In the active region above the $\mathrm{p}$-Well, design rules dictate the existence of Shallow Trench Isolation (STI) between silicon regions. We artificially used these blocks of STI properly choosing their dimensions and positions, to realize a photonic crystal layer (diffraction grating) providing light-trapping effects, in order to locate the maximum of interferences in the SCR.

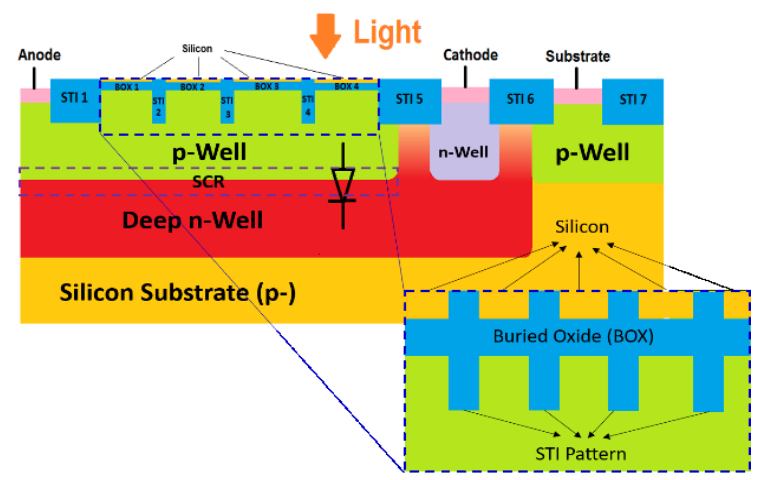

Figure 4 Schematic of SPAD implemented in 28nm FD-SOI CMOS technology (Scales are not respected and BEOL layers are not represented)

From the optical point of view, the SPAD structure could be simplified as shown in Fig. 5. Here the $\mathrm{SiO}_{2}$ region and silicon substrate region are considered semi-infinite.

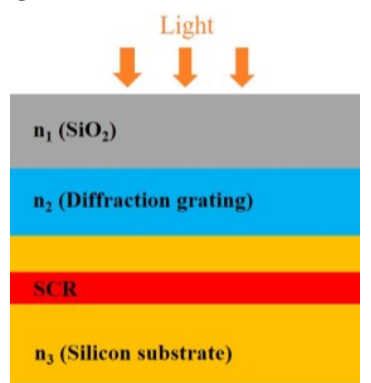

Figure 5 Simplified equivalent optical schematic of SPAD structure

The problematic of improving the photon detection efficiency of SPAD with a diffraction grating comes down to maximizing the light intensity in the SCR (multiplication region). This optimisation is thus realised in two steps. Firstly, the pattern plays the role of an antireflection coating in the case of Front-Side Illumination (FSI) to minimize the reflection coefficient $R_{1}$ at interface $\mathrm{SiO}_{2} /$ diffraction grating. $R_{1}$ is function of the optical index $\mathrm{n}_{1}, \mathrm{n}_{2}$ and $\mathrm{n}_{3}$ and the diffraction grating thickness. The 
equivalent optical refractive index $n_{2, e q}$ in the case of FSI follows a effective medium law [10]:

$$
n_{2, e q}^{2}=n_{\mathrm{SiO}_{2}}^{2} *(1-F F)+n_{\text {silicon }}^{2} * F F
$$

Here the Filling Factor (FF) is defined as the ratio between the silicon area and the total area of one pattern with square shape. The equivalent optical index of diffraction grating layer is thus function of FF. The second step consists of using the interferences of diffraction grating to locate the maximum of light intensity in the SCR where the ATP is the highest. The position of the maximums of interferences is function of the pattern period. Suitable pattern periods allow to improve the photogeneration rate in the SCR and thus to improve the general photon detection probability.

Our simulation methodology is to first find the optimal FF of STI which allows the maximum light transmission by performing optical simulations with a small pattern period (less than $100 \mathrm{~nm}$ ). Once the optimal $F F$ is found, the second step is to extract the optimal spatial period allowing the constructive interferences in the SCR.

\section{Simulation Results}

The simulation methodology was applied to SPAD implemented in 28nm FD-SOI CMOS technology (Fig. 4) in the case of FSI with several constrains. The first one is that the depth of STI blocks is fixed by the technological process. Additionally, the process design rules impose certain restrictions on width, length of STI areas and distance between them. As the SPAD devices are fabricated in standard process, the second constraint refers to Back-end-of-Line (BEOL) layers, which are not optimized for optical light transmission (moreover, their optical properties are not fully characterized). Nevertheless, the optimisation strategy still remains effective.

In the first step, several values of FF were simulated at wavelengths of $500 \mathrm{~nm}$ and 650nm. Fig. 6 and Fig. 7 respectively represent the profiles of electric field magnitude and photogeneration rate with $\mathrm{FF}=0.15$ at wavelength of $500 \mathrm{~nm}$ and with a pattern period of $10 \mathrm{~nm}$. These profiles were extracted near the centre of one pattern and the incident light power was set to $1 \mathrm{~W} / \mathrm{m}^{2}$. The curves showed a significant antireflection effect of pattern with different values of FF compared to reference structure, with

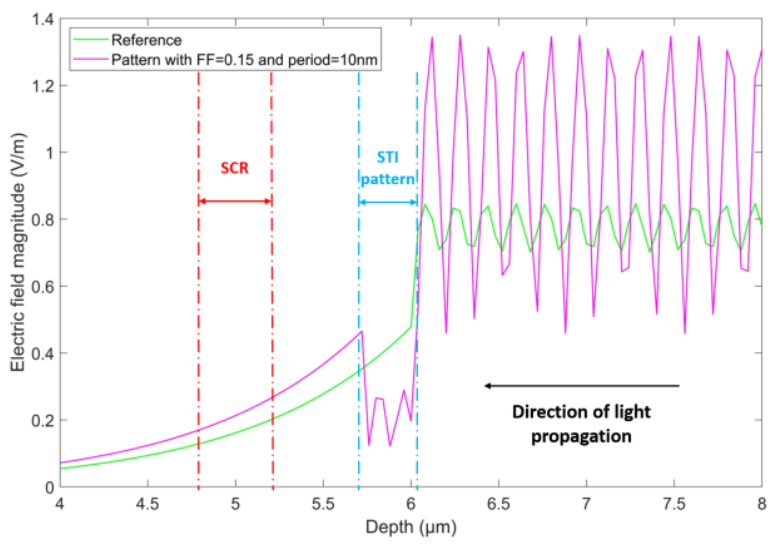

Figure 6 Profiles of electric field magnitude of reference SPAD and patterned $\mathrm{SPAD}$ with $\mathrm{FF}=0.15$ and period $=10 \mathrm{~nm}$

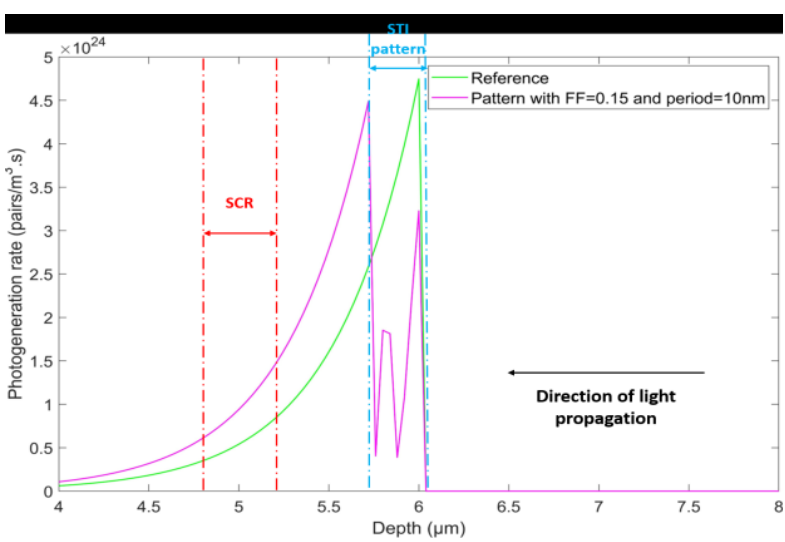

Figure 7 Profiles of photogeneration rate of reference SPAD and patterned $\mathrm{SPAD}$ with $\mathrm{FF}=0.15$ and period $=10 \mathrm{~nm}$

electric field magnitude up to $30 \%$ and of photogeneration rate up to $60 \%$. Considering we didn't fully control the pattern dimensions, the antireflection effect could have been more significant.

The second step was dedicated to the optimisation of pattern period in order to locate the maximums of interferences of pattern grating in the SCR. Several values of pattern period for each FF were performed in simulations. Fig. 8 illustrates a cartography of photogeneration rate at wavelength of $500 \mathrm{~nm}$ with a pattern period of $0.48 \mu \mathrm{m}$ and

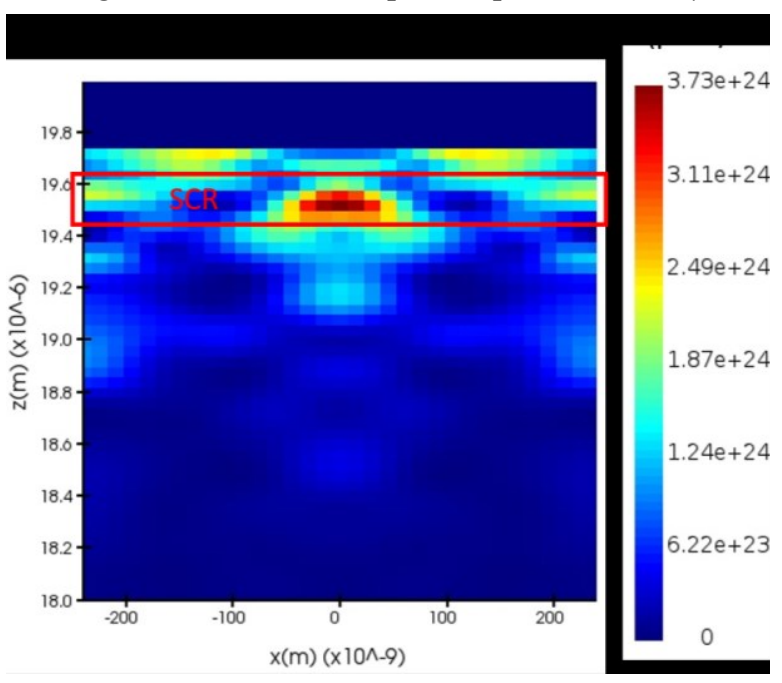

Figure 8 Cartography of photogeneration rate of patterned SPAD with $\mathrm{FF}=0.15$ and period $=0.48 \mu \mathrm{m}$

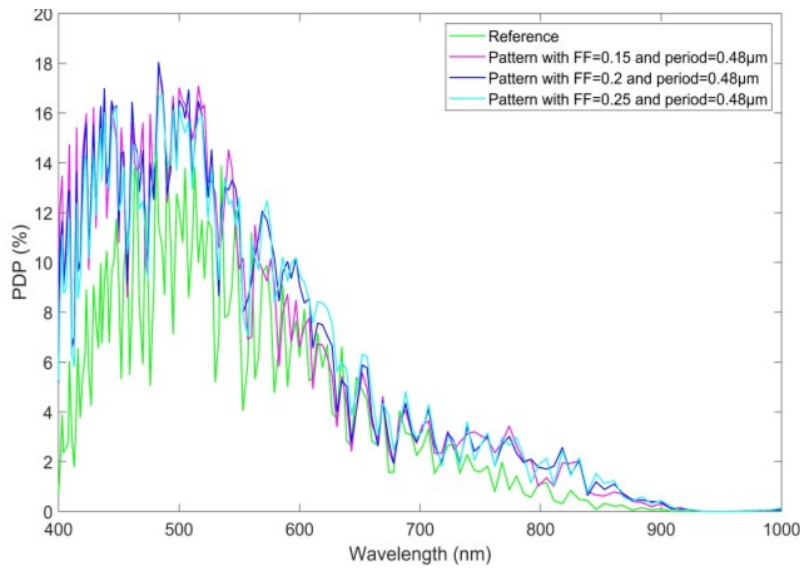

Figure 9 PDP Profiles of reference SPAD and patterned SPAD with excess voltage $\mathrm{V}_{\mathrm{ex}}=0.6 \mathrm{~V}$ 


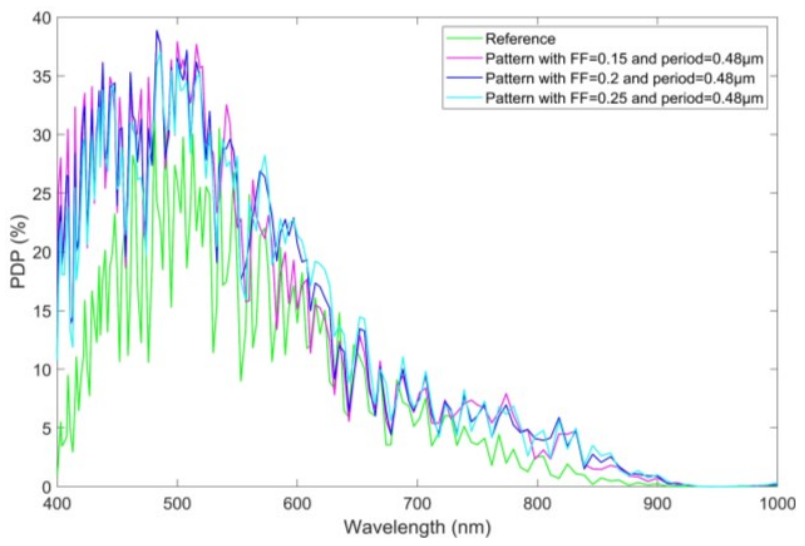

Figure 10 PDP Profiles of reference SPAD and patterned SPAD with excess voltage $\mathrm{V}_{\mathrm{ex}}=1.5 \mathrm{~V}$

an FF of 0.15 . The SCR was located $180 \mathrm{~nm}$ away from the interface diffraction grating/silicon substrate. We could observe the presence of a hot spot in the SCR, showing clearly the diffracting effect of the pattern.

These period dimensions were then used in the simulations with a larger range of wavelengths of 400$1000 \mathrm{~nm}$ in order to investigate the improvement for other wavelengths. Fig. 9 and Fig. 10 respectively illustrate the $P D P$ profiles with an excess voltage $V_{e x}$ of $0.6 \mathrm{~V}$ and $1.5 \mathrm{~V}$ $\left(0.6 \mathrm{~V}\right.$ corresponds to an excess voltage $V_{e x}$ of $6 \%$ of breakdown voltage $V_{b d}$ ). We also chose to work at $V_{e x}=1.5 \mathrm{~V}$, since higher $V_{e x}$ allows higher values ATP of and consequently of $P D P$, thus greater difference between patterning SPAD and reference SPAD. The curves show a clear relative improvement of the $P D P$ for shorter and longer wavelengths. For mid-range wavelengths, the gain in $P D P$ is relatively smaller with higher absolute values of $P D P$ though. The relative gain could reach $700 \%$ at specific wavelengths and the average relative gain could reach over $100 \%$ in the considered range of wavelengths.

Thanks to these simulation results, we have submitted three pattern dimensions for IC fabrication and for future characterization. Table I summarizes the simulated relative improvement of the submitted patterns in different ranges of wavelengths.

Table 1 Simulated relative gain of submitted pattern compared to reference $S P A D$

\begin{tabular}{|c|c|c|}
\hline $\mathrm{FF}$ & $\begin{array}{c}\text { Pattern } \\
\text { period }(\mu \mathrm{m})\end{array}$ & $\begin{array}{l}\text { Average relative gain compared } \\
\text { to reference }\end{array}$ \\
\hline \multirow{3}{*}{0.15} & \multirow{3}{*}{0.48} & $400 \mathrm{~nm}<\lambda<550 \mathrm{~nm}: \sim 140 \%$ \\
\hline & & $550 \mathrm{~nm}<\lambda<750 \mathrm{~nm}: \sim 25 \%$ \\
\hline & & $750 \mathrm{~nm}<\lambda<1000 \mathrm{~nm}: \sim 250 \%$ \\
\hline \multirow{3}{*}{0.2} & \multirow{3}{*}{0.48} & $400 \mathrm{~nm}<\lambda<550 \mathrm{~nm}: \sim 130 \%$ \\
\hline & & $550 \mathrm{~nm}<\lambda<750 \mathrm{~nm}: \sim 30 \%$ \\
\hline & & $750 \mathrm{~nm}<\lambda<1000 \mathrm{~nm}: \sim 340 \%$ \\
\hline \multirow{3}{*}{0.25} & \multirow{3}{*}{0.48} & $400 \mathrm{~nm}<\lambda<550 \mathrm{~nm}: \sim 110 \%$ \\
\hline & & $550 \mathrm{~nm}<\lambda<750 \mathrm{~nm}: \sim 40 \%$ \\
\hline & & $750 \mathrm{~nm}<\lambda<1000 \mathrm{~nm}: \sim 380 \%$ \\
\hline
\end{tabular}

\section{CONCLUSION}

In this article, a 3D electro-optical simulation method for SPAD Photon Detection Probability $(P D P)$ calculation is presented. Then light-trapping concept thanks to the patterning of STI layer is proposed to improve the PDP of a SPAD fabricated in 28nm FD-SOI CMOS technology (with Front Side Illumination). The maximum relative gain of $700 \%$ was reached at specific wavelengths and an average gain of $100 \%$ in the range of wavelengths of 400 $1000 \mathrm{~nm}$ was equally obtained. These encouraging results have permitted IC fabrication including several designs of FD-SOI CMOS SPADs with different STI pattern shapes. Future work will address the characterization of samples with/without an STI pattern and improving the PDP in the case of Back-Side Illumination.

\section{ACKNOWLEDGMENT}

The authors would like to thank the Nano2022 research program for the $\mathrm{PhD}$ Grand of Shaochen Gao, the French national research agency ANR (ANR-18-CE24-0010) for the PhD Grand of Dylan Issartel and CMP (Grenoble) for IC prototyping services.

\section{REFERENCES}

[1] M-J. Lee and E. Charbon, "Progress in single-photon avalanche diode image sensors in standard CMOS: From two-dimensional monolithic to three-dimensional-stacked technology”, Jpn. J. Appl. Phys., vol. 57, no. 1002A3, 2018.

[2] I. Takai, H. Matsunara, M. Soga, M. Ohta, M. Ogawa and T. Yamashita, "Single-Photon Avalanche Diode with Enhanced NIRSensitivity for Automotive LIDAR Systems", Sensors, vol. 16, no. 4, p. 459, 2016.

[3] M.M. Vignetti, F. Calmon, P. Pittet, G. Pares, R. Cellier, L. Quiquerez, T. Chaves de Albuquerque, E. Bechetoille, E. Testa, J-P. Lopez, D. Dauvergne and A. Savoy-Navarro, "3D Silicon Coicidence Avalanche Detector (3D-SiCAD) for charged particle detection", Nucl. Instrum. Methods Phys. Res. A: Accel. Spectrom. Detect. Assoc. Equip, pp. 53-59, 2018.

[4] M. Vitali, D. Bronzi, A.J. Krmpot, S.N. Nilolić, F-J. Schmitt, C. Junghans, S. Tisa, T. Friedrich, V. Vukojević, L. Terenius, F. Zappa and R. Rigler, "A Single-Photon Avalanche Camera for Fluorescence Lifetime Imaging Microscopy and Correlation Spectroscopy”, IEEE J SEL TOP QUANT, vol. 20, no. 6, pp. 344-353, 2014.

[5] T. Chaves De Albuquerque, F. Calmon, R.Clerc, P. Pittet, Y. Benhammou, D. Golanski, S. Jouan, D. Rideau and A. Cathelin, "Integration of SPAD in 28nm FDSOI CMOS technology", $48^{\text {th }}$ European Solid-State Device Research Conference (ESSDERC), pp. 82-85, 2018.

[6] T. Chaves De Albuquerque, D. Issartel, R. Clerc, P. Pittet, R. Cellier, W. Uhring, A. Cathelin and F. Calmon, "Body-biasing considerations with SPAD FDSOI: advantages and drawbacks", 49 $9^{\text {th }}$ European Solid-State Device Research Conference (ESSDERC), pp. 210-213, 2019.

[7] K. Morimoto, "Megapixel SPAD Cameras for Time-Resolved Applications", $\mathrm{PhD}$ thesis, Advanced Quantum Architecture Laboratory, École polytechnique fédérale de Lausanne, Switzerland. http://www.kunter-fonds.ethz.ch/APP_Themes/default/datalinks/Mo orimoto_PhD_Thesis_\%202020.pdf.

[8] L. Frey, M. Marty, S. André and N. Moussy, "Enhancing nearinfrared photodetection efficiency in SPAD with silicon surface nanostructuration", J. Electron Devices Soc., vol. 6, pp. 392-395, 2018.

[9] P. Vines, K. Kuzmenko, J. Kirdoda, D.C.S. Dumas, M.M. Mirza, R.W. Millar, D.J. Paul and G.S. Buller, "High performance planar germanium-on-silicon single-photon avalanche diode detectors", Nat. Commun. vol. 10, no. 1086, 2019.

[10]F. Mandorlo, M. Amara, H.S. Nguyen, A. Charlty-Meano, A. Belarouci and R. Orobtchouk, "Color management of semitransparent nano-patterned surfaces", Opt. Eng., vol. 60(5), p. 055101, 2021 